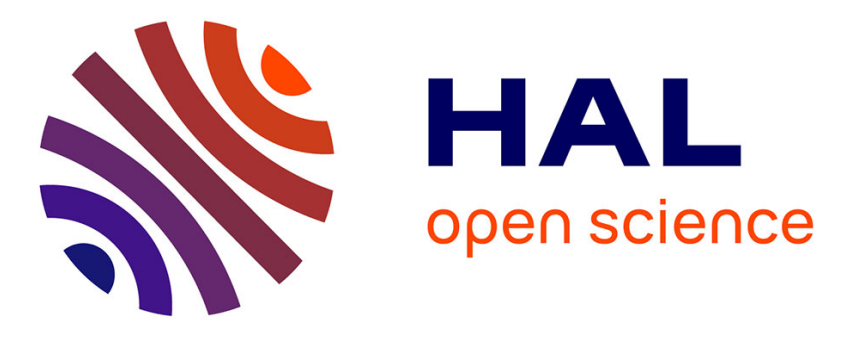

\title{
CK2-regulated schwannomin-interacting protein IQCJ-SCHIP-1 association with AnkG contributes to the maintenance of the axon initial segment
}

Marie-Jeanne Papandréou, Helene Vacher, Marie-Pierre Fache, Esther Klingler, Fanny Rueda-Boroni, Géraldine Ferracci, Claire Debarnot, Christelle Piperoglou, Gontzal Garcia del Caño, Laurence Goutebroze, et al.

\section{To cite this version:}

Marie-Jeanne Papandréou, Helene Vacher, Marie-Pierre Fache, Esther Klingler, Fanny Rueda-Boroni, et al.. CK2-regulated schwannomin-interacting protein IQCJ-SCHIP-1 association with AnkG contributes to the maintenance of the axon initial segment. Journal of Neurochemistry, 2015, 134 (3), pp.527-537. 10.1111/jnc.13158 . hal-01701428

\section{HAL Id: hal-01701428 \\ https://hal.science/hal-01701428}

Submitted on 13 Jul 2018

HAL is a multi-disciplinary open access archive for the deposit and dissemination of scientific research documents, whether they are published or not. The documents may come from teaching and research institutions in France or abroad, or from public or private research centers.
L'archive ouverte pluridisciplinaire HAL, est destinée au dépôt et à la diffusion de documents scientifiques de niveau recherche, publiés ou non, émanant des établissements d'enseignement et de recherche français ou étrangers, des laboratoires publics ou privés. 


\title{
ORIGINAL \\ CK2-regulated schwannomin-interacting protein ARTICLE IOCJ-SCHIP-1 association with AnkG contributes to the maintenance of the axon initial segment
}

\author{
Marie-Jeanne Papandréou, ${ }^{*}$ Hélène Vacher,* Marie-Pierre Fache,* Esther \\ Klingler, $\dagger$ Fanny Rueda-Boroni,* Géraldine Ferracci,* Claire Debarnot,* \\ Christelle Pipéroglou,* Gontzal Garcia Del Caño, $*+$ Laurence Goutebroze $\dagger$ \\ and Bénédicte Dargent* \\ *CRN2M-UMR7286, Aix Marseille Université, CNRS, Marseille, France \\ $\dagger$ Institut du Fer à Moulin, Inserm, UMR-S 839, Université Pierre et Marie-Curie, Paris, France \\ $\ddagger$ Department of Neurosciences, University of the Basque Country, Vitoria-Gasteiz, Spain
}

\begin{abstract}
The axon initial segment (AIS) plays a central role in electrogenesis and in the maintenance of neuronal polarity. Its molecular organization is dependent on the scaffolding protein ankyrin (Ank) $G$ and is regulated by kinases. For example, the phosphorylation of voltage-gated sodium channels by the protein kinase CK2 regulates their interaction with AnkG and, consequently, their accumulation at the AIS. We previously showed that IQ motif containing J-SchwannominInteracting Protein 1 (IQCJ-SCHIP-1), an isoform of the SCHIP-1, accumulated at the AIS in vivo. Here, we analyzed the molecular mechanisms involved in IQCJ-SCHIP-1-specific axonal location. We showed that IQCJ-SCHIP-1 accumulation in the AIS of cultured hippocampal neurons depended on AnkG expression. Pull-down assays and surface plasmon resonance analysis demonstrated that AnkG binds to CK2-
\end{abstract}

phosphorylated IQCJ-SCHIP-1 but not to the non-phosphorylated protein. Surface plasmon resonance approaches using IQCJ-SCHIP-1, SCHIP-1a, another SCHIP-1 isoform, and their $\mathrm{C}$-terminus tail mutants revealed that a segment including multiple CK2-phosphorylatable sites was directly involved in the interaction with AnkG. Pharmacological inhibition of CK2 diminished both IQCJ-SCHIP-1 and AnkG accumulation in the AIS. Silencing SCHIP-1 expression reduced AnkG cluster at the AIS. Finally, over-expression of IQCJ-SCHIP-1 decreased AnkG concentration at the AIS, whereas a mutant deleted of the CK2-regulated AnkG interaction site did not. Our study reveals that CK2-regulated IQJC-SCHIP-1 association with AnkG contributes to AIS maintenance.

Keywords: ankyrin $\mathrm{G}$, axon initial segment, protein kinase CK2, SCHIP-1.

J. Neurochem. (2015) 134, 527-537.
The axon initial segment (AIS) is a highly specialized axonal region that plays a key role in the physiology of mammalian neurons. The AIS not only contains high densities of voltagegated sodium $\left(\mathrm{Na}_{\mathrm{v}}\right)$ and potassium $\left(\mathrm{K}_{\mathrm{v}}\right)$ channels responsible for the action potential firing, but it is also critical for the maintenance of the axonal identity (Leterrier and Dargent 2014). These roles are sustained by the unique molecular architecture of the AIS, which is driven by the scaffolding protein, ankyrin (Ank) G. AnkG is the earliest protein to be targeted to the AIS where it interacts with the actin cytoskeleton via $\beta \mathrm{IV}$-spectrin (Komada 2002) and with microtubules through EB1/3 proteins (Leterrier et al. 2011). AnkG also associates with $\mathrm{Na}_{\mathrm{v}} 1.1, \mathrm{Na}_{\mathrm{v}} 1.2$ and $\mathrm{Na}_{\mathrm{v}} 1.6$, and KCNQ2/3
Received February 28, 2015; revised manuscript received April 14, 2015; accepted May 4, 2015.

Address correspondence and reprint requests to Bénédicte Dargent, CRN2M-UMR7286, Aix Marseille Université, CNRS, Faculté de Médecine-Nord, Boulevard Pierre Dramard, 13015 Marseille, France. E-mail: benedicte.dargent@univ-amu.fr

Abbreviations used: Ab, antibody; AIS, axon initial segment; Ank, ankyrin; API, axonal polarity index; CaM, calmodulin; cdk, cyclindependent kinase; cdk, cyclin-dependent kinase; DIV, day in vitro; DMAT, 2-dimethylamino-4,5,6,7-tetrabromo-1H-benzimidazole; FEZ, fasciculation and elongation protein zeta; GSK, glycogen-synthase kinase; IQCJ-SCHIP-1, IQ motif containing J-Schwannomin-Interacting Protein $1 ; \mathrm{K}_{\mathrm{v}}$, voltage-gated potassium channel; $\mathrm{Na}_{\mathrm{v}}$, voltage-gated sodium channel; Nf-186, neurofascin 186; SPR, surface plasmon resonance; TBB, 4,5,6,7-tetrabromobenzotriazole. 
channels and concentrates them to the AIS (Garrido 2003; Lemaillet 2003; Pan 2006). AnkG binds to the membrane protein neurofascin 186 (Nf-186) that interacts with extracellular matrix proteins (Hedstrom et al. 2007). The crucial role of AnkG in orchestrating the AIS assembly and maintenance was demonstrated by in vivo or in vitro depletion of AnkG. Both depletions prevented AIS formation or dismantled the mature AIS and caused axons to acquire the molecular characteristics of dendrites (Jenkins 2001; Hedstrom et al. 2007, 2008; Sobotzik et al. 2009). During development, AnkB together with $\alpha$ II- and $\beta$ II-spectrin form an intra-axonal boundary limiting AnkG incorporation into the distal axonal submembranous cytoskeleton and eventually causes its accumulation at the proximal axon (Galiano et al. 2012).

We previously showed that IQ motif containing J-Schwannomin-Interacting Protein 1 (IQCJ-SCHIP-1), an isoform of SCHIP-1, is also highly enriched at the AIS in vivo (Martin et al. 2008). Its accumulation at the AIS depends on the presence of AnkG and $\beta I V$ spectrin (Martin et al. 2008) but the functional role of IQCJ-SCHIP-1 is still unravelled. Six isoforms produced by alternative splicing of the Schipl gene are expressed in mouse brain (SCHIP-1a, SCHIP-1b, and IQCJ-SCHIP-1 (Goutebroze et al. 2000; Kwaśnicka-Crawford et al. 2006); SCHIP-1c, SCHIP-1d, and IQCJs-SCHIP-1, GenBank accession numbers KM233716, KM233715 and KJ941154). SCHIP-1a and SCHIP-1b were firstly identified by yeast two-hybrid screen as binding partners of schwannomin (Goutebroze et al. 2000). IQCJ-SCHIP-1 was originally described as a brain protein potentially involved in language disorder in human (Kwaśnicka-Crawford et al. 2006). All SCHIP-1 isoforms share a C-terminal sequence of 234 amino acid residues that contains a leucine zipper coiled-coil domain implicated in their dimerization (Goutebroze et al. 2000). The N-terminal part of IQCJ-SCHIP-1 possesses an IQ motif and associates with calmodulin in the absence of $\mathrm{Ca}^{++}$(Bähler and Rhoads 2002; Martin et al. 2008). This motif is present in IQCJ-SCHIP-1 isoform but absent from the others isoforms. The coiled-coil domain of SCHIP-1 presents about $70 \%$ of conserved residues with one of the four coiled-coil domains of fasciculation and elongation proteins zeta and their Caenorhabditis elegans ortholog (Goutebroze et al. 2000). Fasciculation and elongation proteins zeta proteins are involved in vesicular transport in axons in association with the motor protein kinesin 1 (Bloom and Horvitz 1997; Gindhart et al. 2003; Blasius et al. 2007; Fujita et al. 2007; Ikuta et al. 2007). Moreover, the C-terminal part of SCHIP-1 proteins contains a domain of about 50 amino acids, including 12 serines and threonines phosphorylatable by CK2 (Fig. 2a) (CK2-phosphorylation sites predicted, using GPS 2.0 application, Xue et al. 2008). Interestingly, protein kinase CK2 was found to be enriched at the AIS in vivo and in cultured hippocampal neurons, where it phosphorylated key serine residues of the ankyrin-binding motif of $\mathrm{Na}_{\mathrm{v}} 1.2$ and $\mathrm{Na}_{\mathrm{v}} 1.6$ channels, influencing both $\mathrm{AnkG}$ and $\mathrm{Na}_{\mathrm{v}}$ accumulation at the
AIS and neuronal excitability (Brechet et al. 2008; Brachet et al. 2010; Gasser et al. 2012; Hien et al. 2014).

In this study, we examined whether $\mathrm{CK} 2$ and AnkG are involved in the accumulation of IQCJ-SCHIP-1 at the AIS. We demonstrated that CK2-phosphorylation regulated IQCJSCHIP-1 association with AnkG. The comparison of AnkGbinding properties of IQCJ-SCHIP-1, SCHIP-1a and of their C-terminal tail mutants showed that CK2-phosphorylation domain was directly involved in the interaction with AnkG. Pharmacological inhibition of CK2 diminished both IQCJSCHIP-1 and AnkG accumulation in the AIS. Silencing SCHIP-1 expression reduced AnkG clustering at the AIS. Finally, over-expression of IQCJ-SCHIP-1 decreased AnkG concentration at the AIS, whereas a mutant deleted of the CK2-regulated AnkG interaction site did not. Our study reveals that CK2-regulated IQJC-SCHIP-1 association with AnkG contributes to AIS maintenance.

\section{Experimental procedures}

\section{Animals}

All procedures were in agreement with the European Communities Council directive (86/609/EEC). Experiments were performed on pregnant females Wistar rat or Swiss mice (Janvier labs, Laval, France). Animals were sacrificed by decapitation and embryo brains were used for primary neuronal cell culture.

Plasmids, recombinant protein expression and purification

DNA containing sequence for shRNA against AnkG (Hedstrom et al. 2007) or Firefly luciferase (SHC007; Sigma-Aldrich, SaintLouis, MO, USA) together with a nine nucleotides loop region (TTCAAGAGA) and flanked by $\mathrm{H} 1$ and $\mathrm{U} 6$ promoters were chemically synthesized and inserted into $\mathrm{pCR}^{\mathrm{R}} 2.1$-TOPO (Eurofins MWG/operon, Eberberg, Germany). DNA containing sequence for shRNA against SCHIP-1 (5'-TGCCTCACATAAGCGAATGTT- ${ }^{\prime}$ ) together with a nine nucleotides loop region (TTCAAGAGA) and flanked by nucleotides for BbsI restriction sites was chemically synthesized and subcloned into the double-promoter pFIV-H1/U6 siRNA cloning vector (System Biosciences, Ebersberg, Germany). The efficiency of the resulting vector was verified by performing western blot on lysates from COS-7 cells cotransfected with an expressing vector for Flag-tagged IQCJ-SCHIP-1 (vector pFLAGCMV-2; Sigma-Aldrich) (data not shown). shRNA expression cassettes were then subcloned into EcoR1/Bst $\mathrm{BI}$ of the modified pFUGW vector (Lois 2002) where the EGFP was replaced by farnesylated enhanced green fluorescent protein (EGFP) (from pEGFP-F; Clontech, Rockville, MD, USA). The human nucleotide sequence of SCHIP-1a was amplified by PCR (Expand High Fidelity Taq Polymerase; Roche Molecular Biochemicals, Indianapolis, IN, USA) on pEGFPC3-SCHIP-1a. PCR products were inserted into pCRII-TOPO (TOPO TA Cloning; Life Technologies, Grand Island, NY, USA) and subcloned by restriction into pGEX6P1 (GE Healthcare, Life Science, Little Chalfont, UK). The resulting plasmid (pGEX-SCHIP-1a) drives the expression of GSTSCHIP-1a. To generate the truncated GST-SCHIP-1a mutants, desired restriction sites were introduced on pGEX-SCHIP-1a using Quick Change XL site mutagenesis kit (Stratagene, La Jolla, CA, 
USA). Plasmids expressing mutants were further obtained by enzymatic deletion of the desired region on modified pGEX-SCHIP1a vector. Human nucleotide sequence specific for IQCJ-SCHIP-1 (aa 1-92) flanked by restriction sites was chemically synthesized and inserted into $\mathrm{pCR}^{\mathrm{R}}$ 2.1-TOPO (Eurofin MWG/operon). This sequence was sub-cloned by restriction in the modified pGEXSCHIP-1a to produce pGEX-IQCJ-SCHIP-1. The pFlag-CMV2 IQCJ-SCHIP-1 (Flag-IQCJ-SCHIP-1) was obtained by PCR amplification of the IQCJ-SCHIP-1 cDNA and subcloning into NotI/BglII sites of pFlag-CMV2 vector. The C-terminal tail mutant deleted of its 180 last amino acids (Flag-IQCJ-SCHIP-1- $\Delta$ ter) was generated by subcloning into Not $\mathrm{I} / E c o \mathrm{RV}$ sites of the pFlag-CMV2 vector. All constructs were verified by DNA sequencing (Beckman Coulter Genomics, Danvers, MA, USA). Plasmid expressing GST$\mathrm{Na}_{\mathrm{v}} 1.2_{1080-1203}$ was described in (Brechet et al. 2008). Plasmid expressing rat $270 \mathrm{kDa}-A n k G-G F P$ (Green Fluorescent Protein), purified histidine-tagged human $220 \mathrm{kDa}-\mathrm{AnkB}$ and purified rat $190 \mathrm{kDa}-$ AnkG (Zhang and Bennett 1998) were kindly provided by Dr V. Bennett, (Duke University, Durham, NC, USA). All GSTconstructs were expressed in BL21 Escherichia Coli. Transformed bacteria were grown in M9 minimum medium, supplemented with $3 \%$ ethanol during IPTG induction. GST fusion proteins were purified by chromatography on gluthatione-Sepharose 4B according to the manufacturer's instructions (GE Healthcare). Protein concentrations were determined by the bicinchoninic acid method (Thermo Fisher Scientific, Waltham, MA, USA).

\section{GST pull-downs}

COS-1 cells were transfected with $270 \mathrm{kDa}-A n k G-G F P$ plasmid using Lipofectamine 2000, according to the manufacturer's instructions (Invitrogen, Carlsbad, CA, USA). Transfected cells were solubilized in Tris-buffered saline $(10 \mathrm{mM}$ Tris, $\mathrm{pH} 7.5,150 \mathrm{mM}$ $\mathrm{NaCl}$ ) containing $1 \mathrm{mM}$ EDTA, $1 \%$ Triton X-100, 0.1\% deoxycholate, and protease inhibitors (complete EDTA-free; Roche Molecular Biochemicals) for $45 \mathrm{~min}$ at $4^{\circ} \mathrm{C}$. Insoluble proteins were removed by centrifugation at $14000 \mathrm{~g}$ for $15 \mathrm{~min}$ at $4^{\circ} \mathrm{C}$. GSTproteins $(1 \mu \mathrm{M}$ in $200 \mu \mathrm{L}$ of kinase buffer supplied by the manufacturer) were phosphorylated as previously described in (Brechet et al. 2008) with $200 \mu \mathrm{M}$ ATP (Invitrogen) and $1000 \mathrm{U}$ of CK2 (New England Biolabs, Inc) for $30 \mathrm{~min}$ at $30^{\circ} \mathrm{C}$. To study the interaction with AnkG, GST-proteins were further incubated with $1 \mathrm{~mL}$ of COS- 1 cell lysate for $4 \mathrm{~h}$ at $4^{\circ} \mathrm{C}$. Glutathione Sepharose 4B $(40 \mu \mathrm{L})$ was then added for 1 additional hour. Alternatively, to test SCHIP-1 interaction with calmodulin, GST-proteins were first incubated with Glutathione Sepharose $4 \mathrm{~B}(40 \mu \mathrm{L})$ for $1 \mathrm{~h}$. Beads were washed four times and were resuspended in Tris-buffered saline supplemented with $\mathrm{CaCl}_{2}(2 \mathrm{mM})$ or EGTA $(2 \mathrm{mM})$. Purified calmodulin from bovine brain (Calbiochem, San Diego, CA, USA) ( $2 \mu \mathrm{M}$ in $250 \mu \mathrm{L}$ ) was further added to GST-fusion proteins for 2 additional hours. After extensive washes, pull-down samples were resolved by sodium dodecyl sulfate-polyacrylamide gel electrophoresis and immunoprobed with anti-GST (1/250; Abcam, Cambridge, UK) or anti-calmodulin (1/1000; 05-173; Upstate Biotechnology, Lake Placid, NY, USA) antibodies (Abs).

\section{Surface plasmon resonance}

Surface plasmon resonance (SPR) experiments were performed at $25^{\circ} \mathrm{C}$ on a Biacore 3000 instrument with CM5 sensor chip, using HBSEP running buffer (GE Healthcare) as previously described (Brechet et al. 2008). Briefly, GST or GST-SCHIP-1 proteins were immobilized in flow cells via a polyclonal anti-GST Ab. When indicated, CK2-phosphorylation was directly performed onto the chip according to (Catimel et al. 2006). Purified $190 \mathrm{kDa}-\mathrm{AnkG}$ and $220 \mathrm{kDa}-\mathrm{AnkB}$ were serially diluted two fold in running buffer from 0.5 to $800 \mathrm{nM}$. Samples were injected sequentially at $20 \mu \mathrm{L} / \mathrm{min}$ using the singlecycle kinetic method with an association time of $180 \mathrm{~s}$ and a dissociation time of $60 \mathrm{~s}$, except for the last injection, in which the dissociation time was extended to $15 \mathrm{~min}$ (Karlsson et al. 2006). The non-specific signal (GST) was subtracted from the total signal (GST fusion protein) and control-run injections of buffer were performed in the same conditions before Ank injections and subtracted for data analysis. Binding parameters were measured by globally fitting sensorgrams with the $1: 1$ titration kinetic-binding model with BIAcore evaluation software version 4.1(Palau and Di Primo 2013).

Cell culture, transfection, pharmacological treatment immunofluorescence, and western blot analysis

Primary hippocampal neurons were prepared as described previously (Garrido 2003). Amaxa mice neuron nucleofactor kit (Lonza, Basel, Switzerland) was used to nucleofected dissociated mouse neurons with shRNA against SCHIP-1a or shRNA against luciferase or dissociated rat neurons with shRNA against AnkG or shRNA against luciferase. Mouse neurons were processed for immunofluorescences 7 days after transfection and rat neurons were immunostained 10 days post-tranfection, when all neurons were positive for both AnkG and SCHIP-1 staining. Alternatively, 7 days in vitro (DIV) rat neurons were transfected with Flag-IQCJ-SCHIP-1 or Flag-IQCJ-SCHIP-1- $\Delta$ Cter, using Lipofectamine 2000 (Invitrogen) according to the manufacturer's instructions. Neurons were processed for immunofluorescence 3 days post-transfection. Inhibition of CK2 activity was performed on 9-10 DIV rat neurons, using 4,5,6,7-tetrabromobenzotriazole (TBB, Biaffin GmbH and CoKG: $5 \mu \mathrm{M}$ in $0.05 \%$ dimethylsulfoxide). Controls included the same amount of dimethylsulfoxide. For immunostaining, neurons were blocked for non-specific binding for $30 \mathrm{~min}$ in blocking buffer ( $0.22 \%$ gelatin, $0.066 \%$ saponin in $0.1 \mathrm{M}$ phosphate buffer) after fixation in methanol $\left(7 \mathrm{~min},-20^{\circ} \mathrm{C}\right)$. Proteins were probed with anti-SCHIP (1/100; 17141 directed against the sequence 112-305 of SCHIP-1a, 15013 directed against the sequence 1-97 of IQCJSCHIP-1, Martin et al. 2008), anti-AnkG (1/400; N106/65 and N106/36; from UC Davis/NIH NeuroMab Facility), anti-microtubule-associated protein 2 (MAP2, 1/2000; ab5392; Abcam), antiCK2 (1/50, sc6479; Santa Cruz Biotechnology, Santa Cruz, CA, USA), anti-Nf-186 (1/300, A12/18; NeuroMab), and anti-GFP (1/ 5000, ab 290; Abcam) Abs for $1 \mathrm{~h}$. Corresponding secondary Abs conjugated to Alexa 488, 555, 633 (Invitrogen) or to Dylight 405 (Rockland, Limerick, PA, USA) were incubated for $1 \mathrm{~h}$. Coverslips were mounted in FluorSave reagent (Calbiochem). For western blot analysis, rat neurons cultured from 7 to 21 DIV were solubilized in $10 \mathrm{mM}$ phosphate buffer $\mathrm{pH} 7.4,150 \mathrm{mM} \mathrm{NaCl}, 0.3 \%$ sodium dodecyl sulfate. Protein levels were normalized against $\beta$-tubulin before western blot analysis. Anti-SCHIP-1 Abs (1:200) and anti$\beta$-tubulin Ab (1/1000, clone E7; Developmental Studies Hybridoma Bank, University of Iowa, Iowa, USA) were used for immunoblots.

\section{Confocal microscopy and quantification}

Immunostained neurons were imaged using a Zeiss LMS780 (Zeiss, Jena, Germany) confocal microscope equipped with a 
$63 \times 1.4$ N.A. oil immersion objective. Fluorescence was collected as $\mathrm{Z}$ stacks with sequential wavelength acquisition. Quantification was performed using NIH imageJ software (National Institute of Health, Bethesda, MD, USA). To determine the effect of shRNAs expression, protein immunostaining intensity in the AIS was measured in transfected and in surrounding non-tranfected cells and was expressed as an AIS intensity ratio. Similar measurements were done to examine the impact of IQCJ-SCHIP-1 over-expression. The axonal polarity index i.e., the ratio of average fluorescent intensity for the AIS to dendrite branches, was used to determine the effect of TBB treatments on the localization of AIS proteins. Regions of interest corresponding to AIS were manually selected on AnkG images and reported on other channels for intensity measurements. All intensities were corrected for background labelling. For illustration, image editing was performed using ImageJ or Photoshop 7.0 software (Adobe) and was limited to rolling-ball background subtraction, linear contrast enhancement, and gamma adjustment.

\section{Statistical analysis}

Results are expressed as mean \pm SEM. Statistical analysis was performed using PRISM 5 (Graphpad software, La Jolla, CA, USA). Significance of the differences was assessed using a two-tailed $t$-test: $* * * p<0.0001$.

\section{Results}

Effect of AnkG silencing on IQCJ-SCHIP-1 accumulation at the AIS of hippocampal neurons

We used two anti-SCHIP-1 antibodies (Abs) characterized previously (Martin et al. 2008) to evaluate the expression of SCHIP-1 in hippocampal neurons maintained in culture. The $17141 \mathrm{Ab}$ recognized an epitope present only in SCHIP-1a and in IQCJ-SCHIP-1 and did not show any reactivity for the shorter form, SCHIP-1b (Martin et al. 2008). The $15013 \mathrm{Ab}$ was directed against the first 97 amino acids of IQCJ-SCHIP1, which are specific to this isoform (Fig. 2a). We performed western blot analyses on lysates of neurons collected at different days of culture. Western blotting with either 17141 or 15013 Abs revealed a single protein with an apparent molecular weight of about $65 \mathrm{kDa}$ (Fig. 1a and b), corresponding to IQCJ-SCHIP-1. Accordingly, the AIS of 10 DIV neurons, identified by AnkG labeling, were positive for SCHIP-1 staining by both anti-SCHIP-1 Abs (Fig. 1c and d). AnkG knockdown has been shown to perturb the location of all tested AIS constituents (e.g., Nf-186, $\mathrm{Na}_{\mathrm{v}} 1$ channels and $\beta I V$-spectrin) and to affect proximal axon molecular identity (Hedstrom et al. 2008). Thus, we next evaluated the effect of AnkG depletion on IQCJ-SCHIP-1 accumulation at the AIS. Cultured hippocampal neurons were transfected with farnesylated-GFP shRNA against AnkG (shAnkG) or against luciferase (shcontrol), used as a control (Fig. 1e-g). AnkG immunofluorescent intensity was strongly reduced in shAn$\mathrm{kG}$-transfected neurons as compared to what was observed in shcontrol neurons (ratio of AIS intensity in the transfected neuron to that in the surrounding non-transfected cells,
$0.33 \pm 0.03$ for shAnkG $(n=19), 0.93 \pm 0.07$ for shcontrol $(n=16) ; 2$ independent experiments). This was accompanied by a decrease in IQCJ-SCHIP-1 concentration in the AIS (ratio of AIS intensity $0.31 \pm 0.04$ for shAnkG, $0.92 \pm 0.08$ for shcontrol). We concluded that AnkG is necessary for the recruitment and/or for the retention of IQJC-SCHIP-1 to the AIS.

Protein kinase CK2-phosphorylation regulated IQCJ-SCHIP1 binding to AnkG

To characterize the molecular mechanisms by which IQCJSCHIP-1 concentrates at the AIS, we next subjected cell lysates from COS-1 cells expressing $270 \mathrm{kDa}-\mathrm{AnkG}-\mathrm{GFP}$ to GST pull-down assays with IQCJ-SCHIP-1 or with the canonical SCHIP-1a. As shown in Fig. 2(b) and (c), neither GST-IQCJ-SCHIP-1 nor GST-SCHIP-1a were able to bind to AnkG, unlike GST-Na $1.2_{1080-1203}$ used as a positive control. We previously demonstrated that the phosphorylation of GST-Na $1.2_{1080-1203}$ by CK2 greatly enhanced its interaction with AnkG (Brechet et al. 2008). Since both SCHIP-1 proteins possess a domain predicted as phosphorylatable by this kinase (Fig 2a), we examined if CK2phosphorylation of SCHIP-1 was necessary for its interaction with AnkG. As expected (Brechet et al. 2008; Hien et al. 2014), the binding of AnkG-GFP to GST-Na $1.2_{1080-1203}$ was greatly enhanced upon CK2-phosphorylation (Fig. 2b). Both GST-IQCJ-SCHIP-1 and GST-SCHIP-1a were able to interact with AnkG-GFP after their phosphorylation by CK2 (Fig. 2b and c). In parallel, we wondered whether CK2phosphorylation could also impact IQCJ-SCHIP-1 binding to calmodulin. We tested the ability of GST-IQCJ-SCHIP-1 phosphorylated or not by $\mathrm{CK} 2$, to associate with purified calmodulin in the presence of either $\mathrm{CaCl}_{2}$ or EGTA. GSTSCHIP-1a was used as a negative control. We observed that calmodulin associated with both phosphorylated and nonphosphorylated forms of IQCJ-SCHIP-1 in the absence of $\mathrm{Ca}^{2+}$ (Fig. 2d). Altogether, these results demonstrated that CK2-phosphorylation regulates IQCJ-SCHIP-1 binding to AnkG but not calmodulin association. Moreover, these data suggested that the N-terminal part of the IQCJ-SCHIP-1 that contains the IQ motif, is not required for AnkG binding.

The phosphorylation domain of SCHIP-1 was involved in the binding to AnkG

We next used SPR technology to quantitatively analyze the interaction between AnkG and IQCJ-SCHIP-1 i.e., to determine the binding properties of IQCJ-SCHIP-1 and its interaction domain to AnkG. GST-IQCJ-SCHIP-1 or GSTSCHIP-1a were purified and immobilized on the sensor surface by immunoaffinity. When sequential increasing concentrations of purified $190 \mathrm{kDa}-\mathrm{AnkG}$ were injected over flow cells, no significant reactivity was observed demonstrating that non-phosphorylated GST-SCHIP-1 proteins did not associate with AnkG, in good agreement with 
(a)

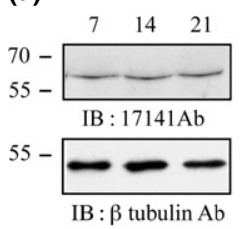

(b)

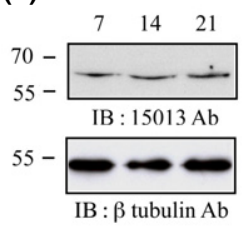

(c)
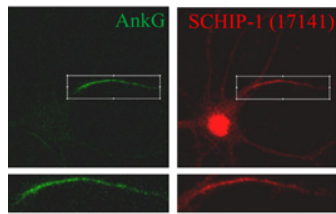

(d)

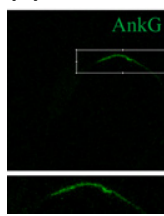

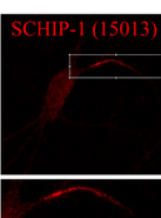
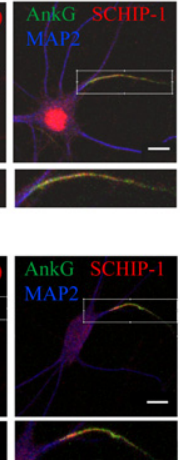

Fig. 1 Effect of ankyrin (Ank) G silencing on IQ motif containing JSchwannomin-Interacting Protein 1 (IQCJ-SCHIP-1) accumulation at the axon initial segment (AIS). ( $a$ and b) Detection of IQCJ-SCHIP-1 expression in cultured hippocampal neurons by western blot. Lysates of neurons cultured from 7 to 21 DIV were analyzed on $12 \%$ sodium dodecyl sulfate-polyacrylamide gel electrophoresis (SDS-PAGE) and immunoblotted (IB) with the 17141 (a) or 15013 (b) Abs as indicated (top panels). Lysates were normalized against tubulin expression (bottom panels). (c and d) Detection of IQCJ-SCHIP-1 expression by

GST pull-down assays (Fig. 3a, b and Table 1). It was only after CK2-phosphorylation performed on immobilized GSTIQJC-SCHIP-1 and GST-SCHIP-1a, that purified AnkG was able to interact with both proteins with an affinity in the nanomolar range $\left(\mathrm{K}_{\mathrm{D}}=0.43 \pm 0.19 \mathrm{nM}\right.$ and $\mathrm{K}_{\mathrm{D}}=$ $0.69 \pm 0.35 \mathrm{nM}$, respectively) (Fig. 3a, b and Table 1). AnkB, which possesses about $70 \%$ of sequence homology with AnkG, is also highly expressed in axons but presents a distinct location and function from AnkG (Galiano et al. 2012). Thus, we wondered if CK2-phosphorylation also regulates SCHIP-1 proteins binding to AnkB. We found that both SCHIP-1 proteins interacted with purified $220 \mathrm{kDa}-$ AnkB, with a nanomolar affinity, solely after their phosphorylation by CK2 (Table 1). We next characterized the AnkG-binding region on SCHIP-1 proteins. Since all SCHIP-1 isoforms differ only in their N-terminal domains, we focused our search on the conserved C-terminal region. We generated three GST fusion proteins fused with (i) the Cterminal moiety of SCHIP-1 (referring as amino acids (aa) 307-487 of SCHIP-1a), (ii) the C-terminus lacking its leucine zipper domain (aa: 307-413), and (iii) the C-terminus lacking the phosphorylation domain (aa: 374-487). Both GST-(307-487) and GST-(307-413) were still able to interact
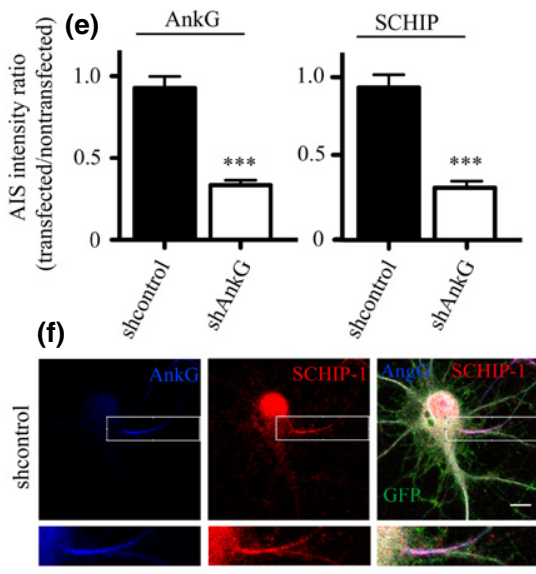

\section{(g)}


immunostaining. 10 DIV neurons were stained with $17141 \mathrm{Ab}$ (red, c) or $15013 \mathrm{Ab}$ (red, d) and Abs to AnkG (green) and MAP2 (blue). Scale bars $=10 \mu \mathrm{m}$. (e-g) Effect of AnkG silencing on AnkG and IQCJSCHIP-1 stainings at the AIS. Neurons transfected with either shluciferase used as a control ( $f$ ) or shAnkG $(g)$ were stained with 17141 (red), anti-GFP (green) and anti-AnkG (blue) Abs. Scale bars $=10 \mu \mathrm{m}$. (e) AIS intensity ratio in the transfected neuron to that in surrounding non-transfected cells for AnkG and IQCJ-SCHIP-1. ${ }^{\star \star *} p<0.0001$

with AnkG after CK2 phosphorylation while GST-(374-487) did not (Fig. 3c and Table 2). These results indicated that the direct interaction between SCHIP-1 proteins and AnkG was mediated through the CK2-phosphorylation domain of SCHIP-1 proteins.

Pharmacological inhibition of CK2 reduced IOCJ-SCHIP-1 and AnkG clustering at the AIS

We tested the impact of the pharmacological inhibition of CK2 on the endogenous IQCJ-SCHIP-1 and AnkG neuronal location. We previously showed that 2-dimethylamino4,5,6,7-tetrabromo-1H-benzimidazole (DMAT), a CK2 inhibitor, reduced both $\mathrm{AnkG}$ and $\mathrm{Na}_{\mathrm{v}}$ concentration at the AIS of mature cultured neurons (Brechet et al. 2008). DMAT is no longer available; we used instead TBB (Duncan et al. 2008). The effect of TBB treatment on the AIS localization of IQCJ-SCHIP-1 and AnkG was analyzed by quantitative immunostaining. The normalized axonal index polarity (API) was determined for AnkG and IQCJ-SCHIP-1 either in TBB-treated cells or in cells treated with the vehicle only (control) (Fig. 4). We observed that TBB treatment $(5 \mu \mathrm{M})$ reduced both IQJC-SCHIP-1 and AnkG accumulation by about $40 \%$ (API $=0.53 \% \pm 0.02$ for IQJC-SCHIP-1 
(a)

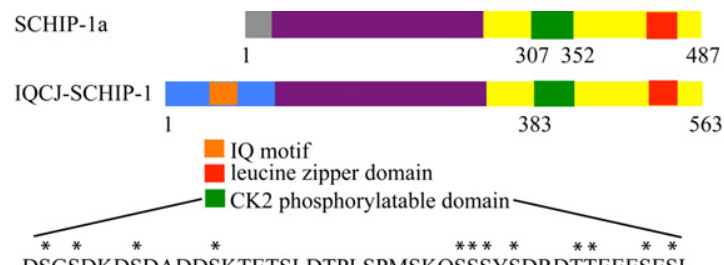

(b)

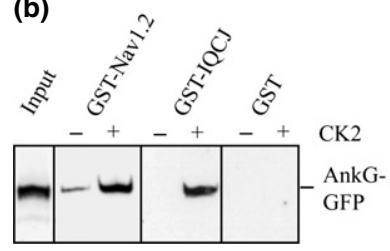

(c)

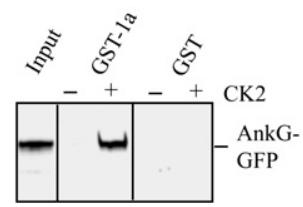

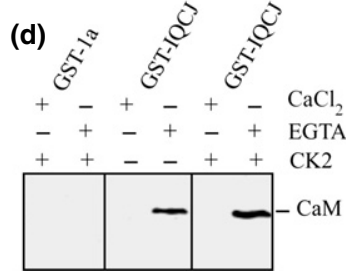

Fig. 2 Effect of CK2 on SCHIP-1 protein association to ankyrin (Ank) $G$ and to calmodulin. (a) Schematic representation of SCHIP-1 isoforms. IQCJ-SCHIP-1 is aligned against SCHIP-1a sequence. Isoforms share a common C-terminal region (yellow box), including a CK2 phosphorylatable domain (green box) and a leucine zipper domain (red box). The N-terminus of SCHIP-1a (gray box) differs with that of IQCJ-SCHIP-1 (blue box), which possesses an IQ motif motif (orange box). The amino-acid sequence of the CK2-phosphorylatable domain is presented; asterisks indicate phosphorylatable Ser and Thr as predicted using GPS 2.0 application (Xue et al. 2008). (b and c) Effect of CK2 on SCHIP-1 protein association with AnkG. GST pulldown reactions were performed on $270 \mathrm{kDa}$-AnkG-GFP (Ank-GFP) lysates using GST-IQCJ-SCHIP-1 (b, GST-IQCJ) and GST-SCHIP-1a (c, GST-1a) in vitro phosphorylated or not by CK2. GST-Na ${ }_{v} 1-2_{1080-}$ 1203 was used as a positive control (b) and GST as a negative one (b and $\mathrm{c}$ ). AnkG-GFP pulled down was detected by western blot with an anti-GFP Ab. (d) Effect of CK2 on IQCJ-SCHIP-1 association with calmodulin (CAM). Purified CAM pull-downs were performed using either non-phosphorylated GST-IQCJ-SCHIP-1 or CK2-phosphorylated GST-IQCJ-SCHIP-1, in the presence $\left(\mathrm{CaCl}_{2}\right)$ or absence (EGTA) of $\mathrm{Ca}^{++}$. CK2-phosphorylated GST-SCHIP-1a was used as negative control. CAM pulled down was detected by immunoblotting with an anti-calmodulin Ab.
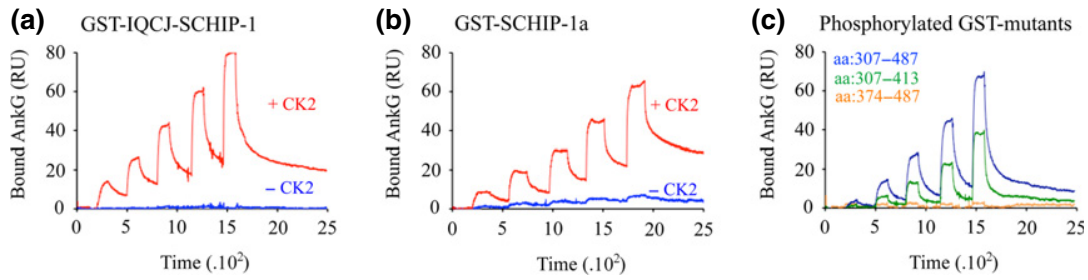

Fig. 3 Analysis of SCHIP-1 protein-binding properties to ankyrin (Ank) $B$ and G. Surface plasmon resonance (SPR) analysis of the direct interaction between SCHIP-1 proteins and AnkG are illustrated by typical sensorgrams. Increasing concentrations of $190 \mathrm{kDa}$-AnkG were injected on immobilized GST-IQCJ-SCHIP-1 (a), GST-SCHIP-

and $0.63 \% \pm 0.03$ for AnkG $(n=115) ; 2$ independent experiments). Altogether these results demonstrated that the accumulation of IQCJ-SCHIP-1 and AnkG at the AIS depended on CK2 activity.

\section{Depletion of IQCJ-SCHIP-1 expression decreased AnkG} clustering in the AIS of hippocampal neurons

Since we previously showed that IQCJ-SCHIP-1 accumulates at the AIS after AnkG (Martin et al. 2008) and that AnkG is required for IQCJ-SCHIP-1 clustering at the AIS (Fig. 1e-g), we further examined whether IQCJ-SCHIP-1 1a (b) before and after in situ phosphorylation by CK2 of the immobilized constructs (c). The position of the amino acids was done from the sequence of SCHIP-1a. Only the curves corresponding to the concentrations of AnkG ranging from 0.5 to $8 \mathrm{nM}$ are shown here.

expression influences the maintenance of the AIS by testing the impact of IQCJ-SCHIP-1 depletion on AnkG clustering. Cultured hippocampal neurons were transfected with validated farnesylated-GFP shRNA against SCHIP-1 (shSCHIP-1) or against luciferase (shcontrol). IQCJ-SCHIP-1 intensity was strongly reduced in shSCHIP-1 transfected neurons as compared to what observed in shcontrol neurons (ratio of IQCJ-SCHIP-1 intensity in the transfected neuron to that in surrounding non-transfected cells, $0.66 \pm 0.04$ for $\operatorname{shSCHIP-1}(n=60), 1.04 \pm 0.04$ for shcontrol $(n=57), 3$ independent experiments). In neurons 
Table 1 Kinetic parameters of the interaction between $190 \mathrm{kDa}-\mathrm{AnkG}$ or $220 \mathrm{kDa}-\mathrm{AnkB}$ and IQCJ-SCHIP-1 or SCHIP-1a

\begin{tabular}{|c|c|c|c|c|c|}
\hline Immobilized GST-construct & Ank & CK2 & $\begin{array}{l}k_{\text {on }} \\
\left(\times 10^{6} \mathrm{M}^{-1} \mathrm{~s}^{-1}\right)\end{array}$ & $\begin{array}{l}k_{\text {off }} \\
\left(\times 10^{-3} \mathrm{~s}^{-1}\right)\end{array}$ & $\begin{array}{l}K_{\mathrm{D}} \\
\mathrm{nM}\end{array}$ \\
\hline \multirow[t]{4}{*}{ IQCJ-SCHIP-1 } & $\mathrm{G}$ & - & & & ND \\
\hline & & + & $2.54 \pm 0.80$ & $0.98 \pm 0.19$ & $0.43 \pm 0.19$ \\
\hline & $\mathrm{B}$ & - & & & ND \\
\hline & & + & $0.66 \pm 0.17$ & $0.32 \pm 0.09$ & $0.46 \pm 0.04$ \\
\hline \multirow[t]{4}{*}{ SCHIP-1a } & $\mathrm{G}$ & - & & & ND \\
\hline & & + & $2.91 \pm 3.14$ & $1.51 \pm 1.27$ & $0.69 \pm 0.35$ \\
\hline & $\mathrm{B}$ & - & & & ND \\
\hline & & + & $0.58 \pm 0.32$ & $0.48 \pm 0.34$ & $0.79 \pm 0.17$ \\
\hline
\end{tabular}

Mean values \pm SD are obtained from 2 to 5 experiments. ND, No sufficient specific binding to determine kinetic parameters and $K_{\mathrm{D}}$.
Table 2 Kinetic parameters of the interaction between 190 kDa-AnkG and SCHIP-1a mutants

\begin{tabular}{|c|c|c|c|c|}
\hline $\begin{array}{l}\text { Immobilized } \\
\text { GST-SCHIP-1a } \\
\text { mutant }\end{array}$ & CK2 & $\begin{array}{l}k_{\mathrm{on}} \\
\left(\times 10^{6} \mathrm{M}^{-1} \mathrm{~s}^{-1}\right)\end{array}$ & $\begin{array}{l}k_{\text {off }} \\
\left(\times 10^{-3} \mathrm{~s}^{-1}\right)\end{array}$ & $\begin{array}{l}K_{\mathrm{D}} \\
\mathrm{nM}\end{array}$ \\
\hline \multirow[t]{2}{*}{ aa: $307-487$} & - & & & ND \\
\hline & + & $3.80 \pm 4.25$ & $1.48 \pm 2.64$ & $2.17 \pm 2.17$ \\
\hline \multirow[t]{2}{*}{ aa: $307-413$} & - & & & ND \\
\hline & + & $2.66 \pm 2.27$ & $9.3 \pm 15.1$ & $2.08 \pm 2.68$ \\
\hline \multirow[t]{2}{*}{ aa: $374-487$} & - & & & ND \\
\hline & + & & & ND \\
\hline
\end{tabular}

Mean values $\pm S D$ are obtained from 2 to 5 experiments. ND, No sufficient specific binding to determine kinetic parameters and $K_{\mathrm{D}}$.

depleted for IQCJ-SCHIP-1, AnkG concentration was also reduced (Fig. 5a-c) (ratio of AIS intensity $0.66 \pm 0.03$ for shSCHIP-1, $0.99 \pm 0.04$ for shcontrol). These results indicated that IQCJ-SCHIP-1 influenced AnkG clustering at the AIS.

\section{Over-expression of IQCJ-SCHIP-1 affected AnkG} accumulation in the AIS of hippocampal neurons To further confirm the role of IQCJ-SCHIP-1 on AIS stability, we transfected cultured hippocampal neurons with either Flag-IQCJ-SCHIP-1 or a C-terminal tail mutant lacking the 180 last amino acids (Flag-IQCJ-SCHIP-1$\Delta$ Cter). Following 3 days of expression, both Flag-IQCJSCHIP-1 and its mutant were expressed throughout the somatodendritic domain identified by MAP2 staining and in axons (Fig. 5d and e). We observed that AnkG clustering was reduced in neurons over-expressing Flag-IQCJ-SCHIP1 , but not in neurons expressing Flag-IQCJ-SCHIP-1- $\Delta$ Cter, the mutant lacking the CK2-regulated AnkG-binding site (ratio of AIS AnkG intensity in transfected cells versus nontransfected cells, $0.50 \pm 0.03$ for Flag-IQCJ-SCHIP-1 $(n=58)$ and $0.99 \pm 0.04$ for Flag-IQCJ-SCHIP-1- $\Delta$ Cter $(n=40) ; 2$ independent experiments (Fig. 5d-f). These results showed that over-expression of IQCJ-SCHIP-1 (a)
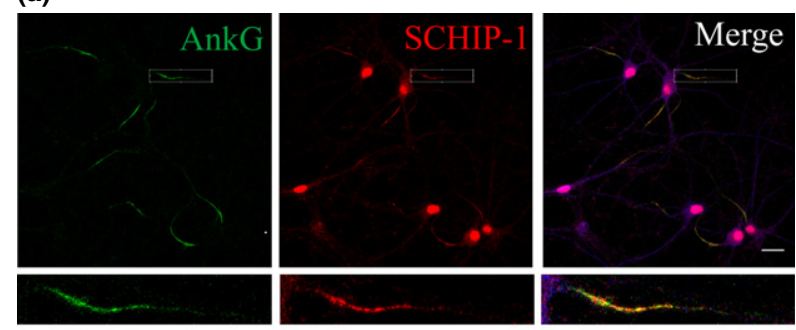

(b)
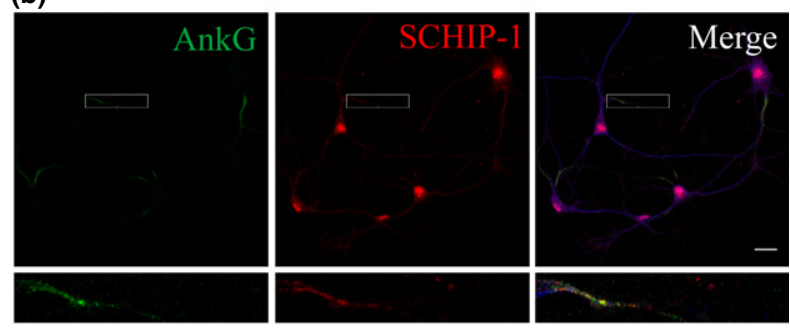

(c)

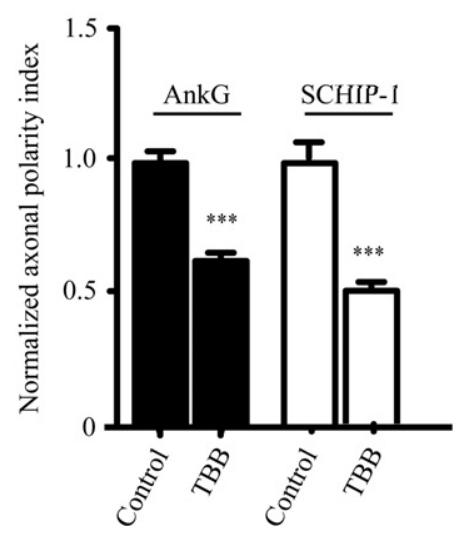

Fig. 4 Effect of CK2 pharmacological inhibition on the axon initial segment (AIS) localization of IQ motif containing J-SchwannominInteracting Protein 1 (IQCJ-SCHIP-1), and ankyrin (Ank) G. (a and b) Cultured hippocampal neurons (9 DIV) treated either with dimethylsulfoxide (DMSO) alone as a control (a) or with $5 \mu \mathrm{M}$ of $4,5,6$, 7-tetrabromobenzotriazole (TBB) (in DMSO) (b) for $24 \mathrm{~h}$ were immunostained for IQCJ-SCHIP-1 and AnkG. Scale bar $=20 \mu \mathrm{m}$. (c) Quantification of IQCJ-SCHIP-1 and AnkG fluorescent intensity. ${ }^{* * *} p<0.0001$ 
(a)
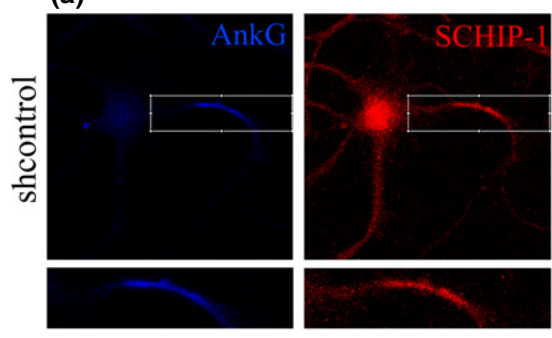

(b)
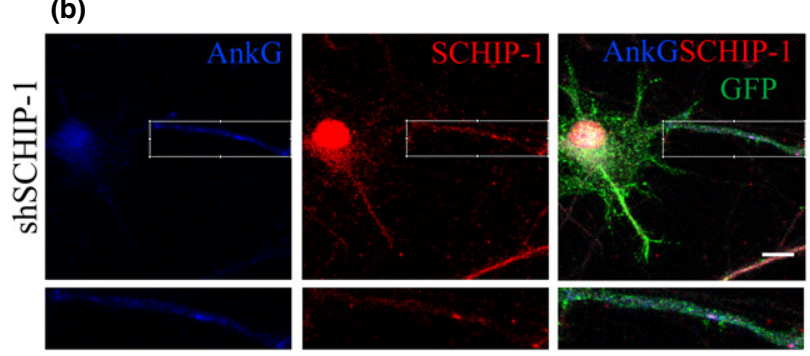

(c)

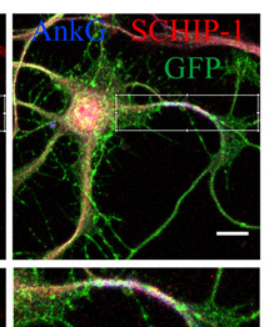

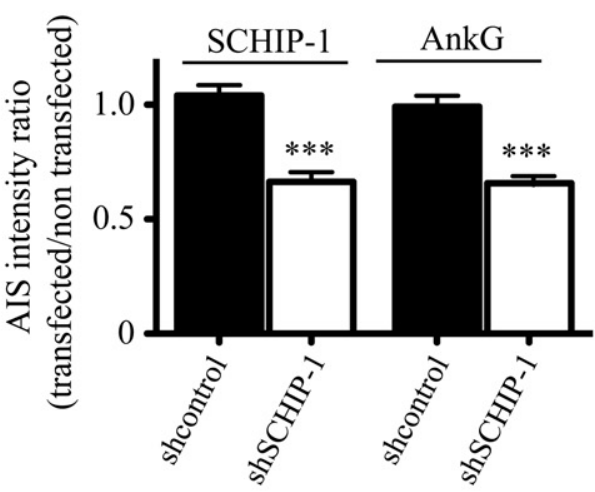

Fig. 5 Effect of IQ motif containing J-Schwannomin-Interacting Protein 1 (IQCJ-SCHIP-1) on ankyrin (Ank) G clustering in the axon initial segment (AIS). (a-c) Effect of IQCJ-SCHIP-1 silencing on IQCJSCHIP-1 and AnkG. Neurons transfected with either shluciferase used as a control (a) or shSCHIP-1 (b) were stained with 17141 (red), antiGFP (green) and anti-AnkG (blue) Abs. Scale bars $=10 \mu \mathrm{m}$. (c) The quantification of the AIS intensity ratio in the transfected neuron to that

reduced endogenous AnkG clustering at the AIS through its CK2-regulated AnkG-binding site.

\section{Discussion}

We previously observed that IQCJ-SCHIP-1 segregated at the AIS of neurons, in vivo (Martin et al. 2008). In this study, we analyzed the molecular mechanisms involved in IQCJ-SCHIP-1-specific axonal location. We showed that IQCJ-SCHIP-1 accumulated at the AIS of hippocampal neurons in culture and that AnkG depletion reduced its concentration. Both pull-down assays and SPR analysis revealed a direct interaction of nanomolar affinity between AnkG and CK2-phosphorylated IQCJ-SCHIP-1 but not with (d)


in surrounding non-transfected cells, for IQCJ-SCHIP-1 and AnkG. (df) Effect of IQCJ-SCHIP-1 over-expression on AnkG. (d and e) Neurons were transfected with Flag-IQCJ-SCHIP-1 (d) and FlagIQCJ-SCHIP-1 $\triangle$ Cter (e) were stained with anti-AnkG (red), anti-GFP (green) and anti-MAP2 (blue) Abs. Scale bars $=10 \mu \mathrm{m}$. (f) AnkG AIS intensity ratio in the transfected neuron to that in surrounding nontransfected cells. ${ }^{* *} p<0.0001$

non-phosphorylated form of the protein. Moreover, we demonstrated that a region of IQCJ-SCHIP-1 including 12 sites phosphorylatable by CK2 was involved in this association. Pharmacological inhibition of CK2 led to a diminution of the IQCJ-SCHIP-1 accumulation at the AIS of cultured neurons. Silencing SCHIP-1 expression reduced AnkG clustering at the AIS. Finally, over-expression of IQCJ-SCHIP-1 in neurons decreased AnkG concentration at the AIS whereas a mutant deleted of the CK2-regulated AnkG interaction site did not. To conclude, our study reveals that CK2-phosphorylation regulates IQJC-SCHIP-1 association with AnkG and consequently its accumulation at the AIS. Our study reveals that CK2-regulated IQJC-SCHIP-1 association with AnkG contributes to AIS maintenance. 
CK2 is a Ser and Thr kinase that has pleitropic effects on a wide range of cellular events. CK2 is thought to be constitutively active and its segregation to specific cell compartments may contribute to its substrate specificity (Montenarh 2010). We previously showed that CK2 was concentrated at the AIS of both cultured neurons and in vivo, and that CK2-phosphorylation of the AnkG-binding motif of $\mathrm{Na}_{\mathrm{v}} 1.2$ and $\mathrm{Na}_{\mathrm{v}} 1.6$ channels not only enhanced channel affinity to AnkG but also contributed to their accumulation at the AIS (Brechet et al. 2008; Brachet et al. 2010; Hien et al. 2014). Here we report that the interaction between IQJC-SCHIP-1 and AnkG is also regulated by $\mathrm{CK} 2$. We observed that the IQ motif of IQCJSCHIP-1 was not required for the in vitro association between AnkG and IQCJ-SCHIP-1 as CK2-phosphorylated SCHIP-1a, which does not possess this IQ motif, still associated with AnkG with a nanomolar-range affinity.

Our SPR experiments revealed that CK2-phosphorylated IQCJ-SCHIP-1 bound to AnkG and to AnkB with equivalent affinities. This result was not really surprising since these two axonal ankyrins present a high degree of sequence homology (Bennett and Healy 2009). However, IQCJ-SCHIP-1 preferentially accumulates at the AIS (AnkG-positive subdomain), but not in distal axons (AnkB-positive subdomain) of cultured hippocampal neurons. In sciatic nerves, IQCJSCHIP-1 proteins co-localized with AnkG in the nodal region of the nodes of Ranvier (Martin et al. 2008), but not in the paranodal regions that contain high concentrations of AnkB (Kordeli et al. 1990; Ogawa 2006). Thus, altogether these data suggest that IQCJ-SCHIP-1 may preferentially interact in vivo with AnkG. One reason for the different results obtained in vivo and in vitro, may be found in the neuronal conformation and position of the axonal ankyrins. Indeed, AnkG is associated with the plasma membrane via a palmitoyl anchor or via its interaction with membrane proteins, while AnkB is essentially expressed as an intracellular protein (He et al. 2012, 2013). Differences in membrane anchoring and/or in ankyrin conformations may sustain that IQCJ-SCHIP-1 binds preferentially to AnkG. It is also tempting to propose that CK2, which is found enriched in both the AIS and the nodes of Ranvier (Brechet et al. 2008), favors only the association between IQCJ-SCHIP-1 and AnkG. This would contribute to IQCJ-SCHIP-1-specific accumulation in only AnkG-positive domains.

Others kinases modulate protein location at the AIS. For example, the cyclin-dependent kinase $2(\operatorname{cdk} 2)$ and $\operatorname{cdk} 5$ kinases regulate $\mathrm{K}_{\mathrm{v}} 1$ channel targeting through the phosphorylation of their auxiliary subunit $\mathrm{K}_{\mathrm{v}} \beta 2$ (Vacher et al. 2011). Furthermore, cdk5 influences protein distribution in the Ank- enriched AIS-like compartment of $\gamma$ neurons, in Drosophila (Trunova et al. 2011). The glycogen-synthase kinase 3 (GSK3) activity was also described to influence both the clustering of AnkG and $\mathrm{Na}_{\mathrm{v}}$ and neuronal excitability of cultured neurons (Tapia et al. 2012). Interestingly, IQCJ-SCHIP-1 also presents several predicted phosphorylation sites for both $\mathrm{cdk} 2 / 5$ and GSK3 kinases (Xue et al. 2008). However, these sites are not located in the CK2-phosphorylatable domain involved in AnkG interaction. Therefore, it is likely that CK2 directs the accumulation of IQCJ-SCHIP-1 at the AIS.

The molecular mechanisms responsible for AnkG retention at the AIS begin to be unraveled. It has been shown that ankyrin B forms an intra-axonal boundary limiting AnkG incorporation into the distal axonal submembranous cytoskeleton and consequently causes its accumulation at the proximal axon (Galiano et al. 2012). Moreover, silencing $\beta I V$-spectrin or neurofascin in knock-out mice or in cultured neurons did not inhibit AnkG segregation at the AIS, (Hedstrom et al. 2008; Le Bras et al. 2013), confirming the role of AnkG as the main organizer of the AIS. On the other hand, we ((Brechet et al. 2008) and present work) and others (Sanchez-Ponce et al. 2011) have shown that the inhibition of CK2 by pharmacological or interference RNA approaches affected AnkG concentration at the AIS, suggesting that AnkG retention at the AIS is also regulated. In the present study, we observed that either depletion of IQCJ-SCHIP-1, or its over-expression, reduced AnkG concentration at the AIS. Moreover, the impact of IQCJ-SCHIP-1 over-expression required the presence of the $\mathrm{C}$-terminal region that carries CK2-regulated AnkG-binding site. Thus, altering the concentration of IQCJ-SCHIP-1 in neurons influences AIS maintenance and this effect depends on CK2-regulated association between IQCJ-SCHIP-1 and AnkG. Altogether, this suggests an interplay between AnkG, IQCJ-SCHIP-1, and CK2 activity: the concentration of AnkG at the AIS would partially depend on its interaction with IQCJ-SCHIP1 , which in turn could be finely controlled by CK2.

\section{Acknowledgments and conflict of interest disclosure}

We thank V. Bennett for providing $270 \mathrm{kDa}-A n k G-G F P$ plasmid, recombinant $220 \mathrm{kDa}-\mathrm{AnkB}$ and $190 \mathrm{kDa}-\mathrm{AnkG}$ proteins; M. Rasband for the generous gift of shRNA against AnkG; Amapola TouatiAutillo and Christophe Leterrier for their help. This work was supported by the Fondation pour la Recherche Médicale grant DEQ20090515387 (to BD) and ANR grant 2011BSV40011 (to BD).

All experiments were conducted in compliance with the ARRIVE guidelines. The authors have no conflict of interest to declare.

\section{References}

Bähler M. and Rhoads A. (2002) Calmodulin signaling via the IQ motif. FEBS Lett. 513, 107-113.

Bennett V. and Healy J. (2009) Membrane domains based on ankyrin and spectrin associated with cell-cell interactions. Cold Spring Harb. Perspect. Biol. 1, a003012.

Blasius T. L., Cai D., Jih G. T., Toret C. P. and Verhey K. J. (2007) Two binding partners cooperate to activate the molecular motor Kinesin1. J. Cell Biol. 176, 11-17. 
Bloom L. and Horvitz H. R. (1997) The Caenorhabditis elegans gene unc-76 and its human homologs define a new gene family involved in axonal outgrowth and fasciculation. Proc. Natl Acad. Sci. USA 94, 3414-3419.

Brachet A., Leterrier C., Irondelle M., Fache M.-P., Racine V., Sibarita J.-B., Choquet D. and Dargent B. (2010) Ankyrin G restricts ion channel diffusion at the axonal initial segment before the establishment of the diffusion barrier. J. Cell Biol. 191, 383-395.

Brechet A., Fache M.-P., Brachet A., Ferracci G., Baude A., Irondelle M., Pereira S., Leterrier C. and Dargent B. (2008) Protein kinase CK2 contributes to the organization of sodium channels in axonal membranes by regulating their interactions with ankyrin G. J. Cell Biol. 183, 1101-1114.

Catimel B., Layton M., Church N., Ross J., Condron M., Faux M., Simpson R. J., Burgess A. W. and Nice E. C. (2006) In situ phosphorylation of immobilized receptors on biosensor surfaces: application to E-cadherin/ $\beta$-catenin interactions. Anal. Biochem. 357, 277-288.

Duncan J. S., Gyenis L., Lenehan J., Bretner M., Graves L. M., Haystead T. A. and Litchfield D. W. (2008) An unbiased evaluation of CK2 inhibitors by chemoproteomics characterization of inhibitor effects on CK2 and identification of novel inhibitor targets. Mol. Cell Proteomics 7, 1077-1088.

Fujita T., Maturana A. D., Ikuta J. et al. (2007) Axonal guidance protein FEZ1 associates with tubulin and kinesin motor protein to transport mitochondria in neurites of NGF-stimulated PC12 cells. Biochem. Biophys. Res. Commun. 361, 605-610.

Galiano M. R., Jha S., Ho T. S.-Y., Zhang C., Ogawa Y., Chang K.-J., Stankewich M. C., Mohler P. J. and Rasband M. N. (2012) A distal axonal cytoskeleton forms an intra-axonal boundary that controls axon initial segment assembly. Cell 149, 1125-1139.

Garrido J. J. (2003) A targeting motif involved in sodium channel clustering at the axonal initial segment. Science 300, 2091-2094.

Gasser A., Ho T. S.-Y., Cheng X., Chang K.-J., Waxman S. G., Rasband M. N. and Dib-Hajj S. D. (2012) An ankyrinG-binding motif is necessary and sufficient for targeting Nav1.6 sodium channels to axon initial segments and nodes of Ranvier. J. Neurosci. 32, 7232-7243.

Gindhart J. G., Chen J., Faulkner M., Gandhi R., Doerner K., Wisniewski T. and Nandlestadt A. (2003) The kinesin-associated protein UNC-76 is required for axonal transport in the Drosophila nervous system. Mol. Biol. Cell 14, 3356-3365.

Goutebroze L., Brault E., Muchardt C., Camonis J. and Thomas G. (2000) Cloning and characterization of SCHIP-1, a novel protein interacting specifically with spliced isoforms and naturally occurring mutant NF2 proteins. Mol. Cell. Biol. 20, $1699-1712$

He M., Jenkins P. and Bennett V. (2012) Cysteine 70 of ankyrin-G Is $S$-palmitoylated and is required for function of ankyrin-G in membrane domain assembly. J. Biol. Chem. 287, 43995-44005.

He M., Tseng W.-C. and Bennett V. (2013) A single divergent exon inhibits ankyrin-B association with the plasma membrane. J. Biol. Chem. 288, 14769-14779.

Hedstrom K. L., Xu X., Ogawa Y., Frischknecht R., Seidenbecher C. I., Shrager P. and Rasband M. N. (2007) Neurofascin assembles a specialized extracellular matrix at the axon initial segment. J. Cell Biol. 178, 875-886.

Hedstrom K. L., Ogawa Y. and Rasband M. N. (2008) AnkyrinG is required for maintenance of the axon initial segment and neuronal polarity. J. Cell Biol. 183, 635-640.

Hien Y. E., Montersino A., Castets F., Leterrier C., Filhol O., Vacher H. and Dargent B. (2014) CK2 accumulation at the axon initial segment depends on sodium channel Nav1. FEBS Lett. 588, 34033408 .
Ikuta J., Maturana A., Fujita T., Okajima T., Tatematsu K., Tanizawa K. and Kuroda S. (2007) Fasciculation and elongation protein zeta-1 (FEZ1) participates in the polarization of hippocampal neuron by controlling the mitochondrial motility. Biochem. Biophys. Res. Commun. 353, 127-132.

Jenkins S. M. (2001) Ankyrin-G coordinates assembly of the spectrinbased membrane skeleton, voltage-gated sodium channels, and L1 CAMs at Purkinje neuron initial segments. J. Cell Biol. 155, 739746.

Karlsson R., Katsamba P. S., Nordin H., Pol E. and Myszka D. G. (2006) Analyzing a kinetic titration series using affinity biosensors. Anal. Biochem. 349, 136-147.

Komada M. (2002) betaIV-spectrin regulates sodium channel clustering through ankyrin-G at axon initial segments and nodes of Ranvier. J. Cell Biol. 156, 337-348.

Kordeli E., Davis J., Trapp B. and Bennett V. (1990) An isoform of ankyrin is localized at nodes of Ranvier in myelinated axons of central and peripheral nerves. J. Cell Biol. 110, 1341-1352.

Kwaśnicka-Crawford D. A., Carson A. R. and Scherer S. W. (2006) IQCJ-SCHIP1, a novel fusion transcript encoding a calmodulinbinding IQ motif protein. Biochem. Biophys. Res. Commun. 350, 890-899.

Le Bras B., Fréal A., Czarnecki A., Legendre P., Bullier E., Komada M., Brophy P. J., Davenne M. and Couraud F. (2014). In vivo assembly of the axon initial segment in motor neurons. Brain Struct. Funct. 219, 1433-1450.

Lemaillet G. (2003) Identification of a conserved ankyrin-binding motif in the family of sodium channel subunits. J. Biol. Chem. 278, 27333-27339.

Leterrier C. and Dargent B. (2014) No Pasaran! Role of the axon initial segment in the regulation of protein transport and the maintenance of axonal identity. Semin. Cell Dev. Biol. 27, 44-51.

Leterrier C., Vacher H., Fache M.-P., d' Ortoli S. A., Castets F., AutilloTouati A. and Dargent B. (2011). End-binding proteins EB3 and EB1 link microtubules to ankyrin $\mathrm{G}$ in the axon initial segment. Proc. Natl Acad. Sci. USA 108, 8826-8831.

Lois C. (2002) Germline transmission and tissue-specific expression of transgenes delivered by lentiviral vectors. Science $\mathbf{2 9 5}, 868$ 872.

Martin P.-M., Carnaud M., del Cano G. G., Irondelle M., Irinopoulou T., Girault J.-A., Dargent B. and Goutebroze L. (2008) Schwannomininteracting protein-1 isoform IQCJ-SCHIP-1 is a late component of nodes of Ranvier and axon initial segments. J. Neurosci. 28, 61116117.

Montenarh M. (2010) Cellular regulators of protein kinase CK2. Cell Tissue Res. 342, 139-146.

Ogawa Y. (2006) Spectrins and ankyrinB constitute a specialized paranodal cytoskeleton. J. Neurosci. 26, 5230-5239.

Palau W. and Di Primo C. (2013) Simulated single-cycle kinetics improves the design of surface plasmon resonance assays. Talanta 114, 211-216.

Pan Z. (2006) A common ankyrin-G-based mechanism retains KCNQ and $\mathrm{NaV}$ channels at electrically active domains of the axon. $J$. Neurosci. 26, 2599-2613.

Sanchez-Ponce D., Muñoz A. and Garrido J. J. (2011) Casein kinase 2 and microtubules control axon initial segment formation. Mol. Cell Neurosci. 46, 222-234.

Sobotzik J.-M., Sie J. M., Politi C., Del Turco D., Bennett V., Deller T. and Schultz C. (2009) Ankyring is required to maintain axodendritic polarity in vivo. Proc. Natl Acad. Sci. USA 106, 17564 17569.

Tapia M., Del Puerto A., Puime A. et al. (2012) GSK3 and $\beta$-catenin determines functional expression of sodium channels at the axon initial segment. Cell. Mol. Life Sci. 70, 105-120. 
Trunova S., Baek B. and Giniger E. (2011) Cdk5 regulates the size of an axon initial segment-like compartment in mushroom body neurons of the Drosophila central brain. J. Neurosci. 31, 10451-10462.

Vacher H., Yang J.-W., Cerda O., Autillo-Touati A., Dargent B. and Trimmer J. S. (2011) Cdk-mediated phosphorylation of the Kv 2 auxiliary subunit regulates Kv1 channel axonal targeting. J. Cell Biol. 192, 813-824.
Xue Y., Ren J., Gao X., Jin C., Wen L. and Yao X. (2008) GPS 2.0, a tool to predict kinase-specific phosphorylation sites in hierarchy. Mol. Cell Proteomics 7, 1598-1608.

Zhang X. and Bennett V. (1998) Restriction of 480/270-kD ankyrin G to axon proximal segments requires multiple ankyrin G-specific domains. J. Cell Biol. 142, 1571-1581. 